\title{
Electrical transport and mechanical properties of alkylsilane self-assembled monolayers on silicon surfaces probed by atomic force microscopy
}

\author{
Jeong Young Park ${ }^{1, *}$ Yabing Qi ${ }^{1,2}$, Paul D. Ashby ${ }^{1}$, Bas L. M. Hendriksen ${ }^{1}$, and \\ Miquel Salmeron ${ }^{1,3, *}$ \\ ${ }^{1}$ Materials Sciences Division, Lawrence Berkeley National Laboratory, University of \\ California, Berkeley CA 94720 \\ ${ }^{2}$ Applied Science and Technology Graduate Group, University of California, Berkeley \\ CA 94720 \\ ${ }^{3}$ Materials Science and Engineering Department, University of California, Berkeley CA
} 94720

The correlation between molecular conductivity and mechanical properties (molecular deformation and frictional responses) of hexadecylsilane self-assembled monolayers was studied with conductive probe atomic force microscopy / friction force microscopy in ultrahigh vacuum. Current and friction were measured as a function of applied pressure, simultaneously, while imaging the topography of self-assembled monolayer molecule islands and silicon surfaces covered with a thin oxide layer. Friction images reveal lower friction over the molecules forming islands than over the bare silicon surface, indicating the lubricating functionality of alkylsilane molecules. By measuring the tunneling current change due to changing of the height of the molecular islands by tilting the molecules under pressure from the tip we obtained an effective conductance decay constant $(\beta)$ of $0.52 / \AA$.

*Authors to whom correspondence should be addressed. Electronic mail: jypark@lbl.gov, mbsalmeron@lbl.gov 


\section{Introduction:}

Friction and adhesion are important issues for controlling the efficiency of nanoscale moving parts. Passivating surfaces with self-assembled monolayers (SAMs) is one of the strategies used for minimizing stiction and reducing adhesion and friction in micro- or nano-electromechanical systems (MEMS/NEMS) ${ }^{1-4}$. Due to their robust and inert nature, SAMs with silane head groups chemistry allow for passivation of hydrophilic silicon surfaces and show promise to reduce both adhesion and friction at MEMS interfaces and thus improve device performance ${ }^{1,2} 5$. The tight packing of SAMs due to Van der Waals attractive forces prevents, at moderate loads, low energy excitations (molecular bending or tilt, gauche defects, etc.), which are major contributors to energy dissipation processes during friction ${ }^{4}$, implying the strong correlation between the tribological properties and molecular conformation in the SAMs.

The electronic structures of SAMs with silane head groups have been extensively studied with various techniques such as conductive atomic force microscope (AFM) or mercury-drop junctions with potential application of molecular electronic devices ${ }^{67,8}$. For example, octadecyltrichlorosilane (OTS) has been proposed and used as a gate insulator in organic electronics ${ }^{9}$. Earlier studies indicate that the charge transport properties of SAMs with silane head groups is greatly influenced by the molecular deformation that results in structural variation of molecules such as tilting or disordering ${ }^{10}$.

In this paper we present a study of the charge transport properties of SAMs with silane head groups that has served as a good model lubricant system. Our approach involves the formation of SAM islands with the size of 100-200 nm. The heights of islands (molecular deformation) can be obtained from topographical AFM images, while 
nanotribological and charge transport properties of SAMs are measured using AFM with a conducting tip. In this manner, we can vary the load applied to the tip-sample contact while simultaneously measuring the frictional responses and electronic conductance as illustrated in Fig. 1 and thus investigate the role of molecular deformation or tilting in determining the decay parameter $\beta$.

\section{Experimental details}

The samples consist of islands of alkylsilanols: $\mathrm{CH}_{3}-\left(\mathrm{CH}_{2}\right)_{\mathrm{n}-1}-\mathrm{Si}(\mathrm{OH})_{3}$, with $\mathrm{n}=16$ adsorbed on silicon surface. The compounds, received as triethoxysilanes, were diluted in an ethanoic solution. The size, number, and distribution of islands on the surface depend on immersion time and solution concentration ${ }^{1}$. Pieces of silicon (111) wafer (Virginia Semiconductor, $\mathrm{n}$ type, $0.001 \mathrm{Ohm} . \mathrm{cm}$ ) were dipped in a dilute HF solution to remove the native oxide layer and immediately transferred to pure water for a thin oxide layer regrowth. The wafer was then dipped for $15 \mathrm{~s}$ directly in a $2 \mathrm{mM}$ ethanoic solution of triethoxysilanes, which also contained $\mathrm{HCl}$ in $1 \mathrm{mM}$ concentration. Lastly, the sample was rinsed with ethanol and blown dry with nitrogen gas.

This preparation technique produced monolayer islands of alkylsilane surrounded by clean silicon oxide as opposed to preparations from aprotic solvents which frequently leave a residue of alkylsilane loosely packed and lying horizontally on the silicon oxide surface. We hypothesize that the success of the ethanoic solution is due to the strong binding of the protic solvents to the silicon oxide surface. The solvent protects the surface from deposition of the alkylsilane unless the silane-surface bond is formed. 
The friction experiments were performed with a commercial RHK-Technology STM/AFM system mounted in a chamber with a base pressure of $1.0 \times 10^{-10}$ torr $^{11}$. The use of a vacuum environment is important because in air, even small amounts of water due to the ambient humidity can lead to oxidation reactions of the Si at positive sample bias ${ }^{12}$. We used cantilevers with spring constants of $2.5 \mathrm{~N} / \mathrm{m}$ coated with approximately $30 \mathrm{~nm}$ of TiN, which is hard and conducting. The normal force exerted by the cantilever was maintained constant during AFM imaging, while the current and friction force between tip and sample were recorded simultaneously ${ }^{13,14}$. To determine the forces, the cantilever spring constant was calibrated by using the resonance-damping method of Sader et al., ${ }^{15}$ while the lateral force was calibrated with the wedge method of Ogletree et al. ${ }^{16}$ The radii of the metal-coated tips were $30-50 \mathrm{~nm}$ before contact, as measured by scanning electron microscopy. When measured after a contact experiment however, the radii were found to be $80-120 \mathrm{~nm}$. The loads used in this study were small enough so that no damage was produced on the surface, as determined by inspection of the images with Ångstrom depth sensitivity as well as by the reproducibility of the friction and adhesion measurements. Since the measured friction force did not change at constant load and did not show time-dependent behavior in the elastic regime, we assume that the changes in tip radius took place soon after the first contact, with minimal changes during subsequent contact measurements.

\section{Results}


Figs.2a, 2b, 2c show the topographic, friction, and current images, respectively, that were acquired simultaneously on hexadecylsilane islands. The image size is $500 \mathrm{x} 500$ nm. Figs. 2d, 2e, 2f show the line profiles of topography, friction, and current images, respectively that were measured at the line marked in Fig 2a. As shown in the line profile of topography, the hexadecylsilane islands have the size of 100-200 nm and the height of $1.6 \mathrm{~nm}$ at the applied load of $0 \mathrm{nN}$ (or effective total load of $20 \mathrm{nN}$ ). Friction on the island is approximately three times lower than that on the surrounding bare silicon, consistent with the low friction of the lubricating alkylsilane layer. It is also clear that the current measured on the alkylsilane island is much smaller than that measured on the silicon surface. From the line profile of the current map, the current measured on the silicon surface is $420 \pm 160 \mathrm{nA}$. The high noise level can be associated with electrical character of third bodies between the tip and the sample such as the thin oxide or organic molecules.

Fig. 3 shows the current-voltage (I-V) characteristics of the junction composed of a conductive tip and hexadecylsilane island that was measured for the applied load between -20 and $80 \mathrm{nN}$. The I-V curves measures on the molecular islands are asymmetric, showing high current at negative sample bias and low current at positive bias. This indicates a rectifying effect in an $\mathrm{n}$-type semiconductor, i.e., a negative sample bias leads to accumulation of electrons at the surface, and the current 'turns on' sharply for small values of the bias. At a positive sample bias, the metal-insulator-semiconductor junction is reverse biased and shows depletion at small voltages ${ }^{17} 18,19$. Fig. 3 also shows the I-V curve measured at the silicon surface with the applied load of $0 \mathrm{nN}$. The measurement of current on the molecules is reversible up to the applied load of $40 \mathrm{nN}$. But at the higher 
load, the contact AFM measurement is not reversible, indicating that the molecular islands are deformed irreversibly at high pressures.

Friction and current were measured simultaneously as a function of the applied load on silicon and hexadecylsilane molecules as shown in Fig. 4. This was possible because the tip scanned over both the bare silicon and hexadecylsilane molecular islands in contact mode. Fig. $4 \mathrm{a}$ and $4 \mathrm{~b}$ show plots of friction and current versus applied load, respectively. The inset shows the friction and current maps. In these maps, the tip scans repeatedly along one direction only (the y axis), while the applied load is increased stepwise in each scan line along the $\mathrm{x}$ axis. Friction on the island is approximately three times lower than that on silicon surface at the applied load between -20 and $40 \mathrm{nN}$. In the wearless regime the friction at the single asperity (f) is described by $f=\tau \mathrm{A}$, where $\mathrm{A}$ is the contact area and $\tau$ is shear stress of the interface. The contact area, A depends on the applied load and adhesion force. Since as shown in the Fig. 4a, the adhesion force is similar and equal to $20 \pm 5 \mathrm{nN}$ on both the silicon and the molecular islands, the contact area at the same applied load should also be the same. Therefore, the lower friction force measured on the molecule islands is due to the lower shear stress, which reflects the lubricating nature of alkylsilane self-assembled monolayer. The friction forces on silicon surface exhibit a rather linear dependence. This can be associated with the multiple contact between the AFM tip and silicon covered with the amorphous oxide or nonuniform value of shear stress $(\tau)$ of the interface. As shown in Fig. $4 b$, the current measured on the molecule islands is much lower than that on the silicon surface (by one or two orders of magnitude depending on the applied load). It is also noticeable that the current on the molecule islands increases more rapidly with increasing load than on the 
bare silicon area. While on silicon the current increases in proportion to the contact area ${ }^{18}$, on top of the islands it increases more rapidly. As discussed below this is connected with a tilting of molecules under load, which in addition to introducing changes in the bonding structure between molecule and substrate may open additional tunneling channels through neighboring molecules.

Fig. 5 shows a semilog plot of current density $\left(\mathrm{nA} / \mathrm{nm}^{2}\right)$ as a function of molecular island height. The contact area was calculated using classical models of contact mechanics, specifically the Johnson-Kendall-Roberts (JKR) model ${ }^{20}$. From the JKR model, the contact area (A) is given by, $A=\pi \cdot\left[\left(6 \pi \gamma \mathrm{R}^{2} / \mathrm{K}\right)\right]^{1 / 3} \cdot\left[\left(1-\sqrt{ }\left(1-\mathrm{L} / \mathrm{L}_{\mathrm{c}}\right)\right) / 2\right]^{2 / 3}$, where $\mathrm{L}_{\mathrm{c}}$ is the adhesion force, $\mathrm{R}$ is the tip radius. $\mathrm{K}$ is the combined elastic modulus, given by the equation $\mathrm{K}=4 / 3 \cdot\left[\left(1-v_{1}^{2}\right) / \mathrm{E}_{1}+\left(1-\mathrm{v}_{2}^{2}\right) / \mathrm{E}_{2}\right]^{-1}$ where $\mathrm{E}_{1}$ and $\mathrm{E}_{2}$ are the Young's moduli, and $v_{1}$ and $v_{2}$ are the Poisson ratios of the tip and substrate, respectively ${ }^{21}$. For the estimation of contact area, we used the tip radius of $100 \mathrm{~nm}, \mathrm{~K}_{\mathrm{TiN}-\mathrm{Si}}$ of $140 \mathrm{GPa}$, and adhesion force $\left(\mathrm{L}_{\mathrm{c}}\right)$ of $20 \pm 5 \mathrm{nN}$ that was separately measured from force-distance curves on the molecular islands. As can be seen from Fig. $5, \ln (\mathrm{J})$ decreases linearly with tipsurface separation, with an average slope $\beta=0.52 \pm 0.04 \AA^{-1}$.

\section{Discussion}

According to the commonly used approximation of the Simmons model, the current (I) is expressed), $I=I_{0} \exp (-\beta d)$, where $\beta$ is the characteristic decay parameter for this tunneling junction, and $\mathrm{d}$ is the molecular film thickness (the separation between electrodes). Based on the height of the molecular islands and the measured current at 
each height, a decay constant $\beta$ can be estimated. In the case of alkylthiol self-assembled monolayers on $\mathrm{Au}(111)$, it was shown that the current decreases exponentially with tipsurface separation with an average slope of $0.53-0.57 \AA^{-1} 14,22$. This tunneling decay constant $\beta$ through molecules of fixed chain length is significantly smaller than that for the case where the distance between electrodes is changed by using alkanethiol molecules of different lengths $\left(\beta \sim 1 \AA^{-1}\right)$. Salomon et al. measured current-voltage curves ${ }^{7}$ in n-Si$\mathrm{C}_{\mathrm{n}} \mathrm{H}_{2 \mathrm{n}} / \mathrm{Hg}$ junctions in the voltage range between 0.7 and $1.0 \mathrm{~V}$ (forward bias) as a function of chain length $(n=12,14,16,18)$. From this result a value of $\beta=0.56 \pm 0.04 \AA^{-1}$ was obtained. In contrast, Zhao et al. obtained $\beta=0.75-0.95 \AA^{-1}$ in the voltage range of 3.0 and $3.8 \mathrm{~V}$, upon shortening the chain lengh, using conductive probe AFM on films of $\mathrm{CH}_{2}=\mathrm{CH}-\left(\mathrm{CH}_{2}\right)_{\mathrm{n}-3} \mathrm{CH}_{3}(\mathrm{n}=12,14,16$, and 18$)$ on n-type silicon (111) surface ${ }^{23}$. They also observed an increase of current by almost 3 orders of magnitude as increasing the applied load from 4.4 to $46.2 \mathrm{nN}$, which was mainly attributed to shortening the tunneling distance and partially to the increase in contact area. In that study the tunneling decay constant $\beta$ upon tilting was not determined because the thickness of molecular thickness upon the molecular deformation was not known.

Since in our case the molecular length is the same for all electrode separations, one would expect ideally a value of $\beta$ equal to zero if electron transport were determined purely by tunneling along the alkane chains. Our result of tunneling decay constant $\beta=$ $0.52 \AA^{-1}$ through molecules of fixed chain length is halfway between zero and the value obtained by changing the alkyl chain length $\left(0.56 \AA^{-1}\right.$ by Salomon et al. and $0.75-0.95$ $\AA^{-1}$ by Zhao et al.). 
Slowinski et al. ${ }^{24}$ proposed a two-pathway conductance model involving tunneling along the length of the alkyl chain (through-bond), and also chain-to-chain coupling. The first pathway is independent of tilt, while the second depends on the tilt angle. In this simplified model, the tunneling current is given by:

$$
I_{t}=I_{0} \exp \left(-\beta_{T B} d\right)+I_{0} n_{s} \exp \left[-\beta_{T B}\left(d-d_{C C} \tan \Theta\right)\right] \exp \left(-\beta_{T S} d_{C C}\right)
$$

where $I_{t}$ is the current at a specific tilt angle $\Theta, d$ the length of the molecule (for C16, $d=24 \AA), n_{s}$ is a statistical factor accounting for the number of pathways containing a single lateral hop as compared to those containing only through-bond hops. In our case $n_{s}=16$, the number of carbon atoms in the molecule; $d_{C C}=4.3 \AA$, the diameter of the alkane chains; and $\beta_{T B}$ and $\beta_{T S}$ are respectively through-bond and through-space decay constants. Fig. 5 shows the best fit of two pathway model into the experimental data of current measured as a function of heights of molecule islands by using the fitting parameters of $\beta_{T B}$ and $\beta_{T S}$, that are $0.9 \AA^{-1}$ and $1.1 \AA^{-1}$ respectively. The good fit indicates that the two-path tunneling model is indeed compatible with the observations.

It is perhaps not surprising that such model produces good fits with the results obtained with alkylsilane SAMs, as it did for the alkylthiol SAMs ${ }^{24}$. This is expected from the increased number of adjustable parameters relative to simple tunneling process. Our understanding of the physical processes determining charge transfer and tunneling will be incomplete however until the changes in binding between the head groups versus tilt angle are understood. Indeed it was proposed that changes in S-Au binding of the alkylthiol SAMs due to distortions in the chain structure lead to changes in tunneling characteristics, in addition to changes due to increasing tunneling across molecules. 
Additional conductance channel involves multiple tunneling junctions through multiple molecules. Similar considerations can apply to the binding between the head groups ( $\mathrm{Si}$ $\mathrm{O}-\mathrm{Si}$ and $-\mathrm{OH}$ ), which could also be changed under load and produce changes in the tunneling junction characteristics.

\section{Conclusion}

Influence of charge transport properties on the molecular deformation and frictional responses was investigated for the nanoscale junction of AFM metal coated tip/ alkylsilane/silicon surface. Lower current and friction were observed on the hexadecylsilane islands comparing to the silicon surface. We obtained the effective conductance decay constant $(\beta)$ of $0.52 \AA^{-1}$ upon tilting of molecules. If the two-path model is used to fit the data, through-bond and through-space decay constants of 0.9 (/ $\AA$ ) and $1.1(/ \AA)$ are obtained. An understanding of the tunneling processes through these insulating alkyl-chain organic films requires a better understanding of the changes in binding structure of the head groups to the substrate.

\section{ACKNOWLEDGEMENT}

This work was supported by the Director, Office of Energy Research, Office of Basic Energy Sciences, Molecular Foundry, Materials Sciences Division, U.S. Department of Energy through the Lawrence Berkeley National Laboratory (contract no. DE-AC0205CH11231). 


\section{Reference}

1 E. Barrena, S. Kopta, D. F. Ogletree, D. H. Charych, and M. Salmeron, Physical Review Letters 82, 2880 (1999).

2 B. Bhushan and H. W. Liu, Physical Review B 63, 245412 (2001); E. E. Flater, W. R. Ashurst, and R. W. Carpick, Langmuir 23, 9242 (2007); N. S. Tambe and B. Bhushan, Nanotechnology 16, 1549 (2005); X. D. Xiao, J. Hu, D. H. Charych, and M. Salmeron, Langmuir 12, 235 (1996); L. Z. Zhang, L. Y. Li, S. F. Chen, and S. Y. Jiang, Langmuir 18, 5448 (2002).

O. P. Khatri and S. K. Biswas, Journal of Physical Chemistry C 111, 2696 (2007);

H. Liu and B. Bhushan, Ultramicroscopy 91, 185 (2002).

M. Salmeron, Tribology Letters 10, 69 (2001).

5

S. Onclin, B. J. Ravoo, and D. N. Reinhoudt, Angewandte Chemie-International Edition 44, 6282 (2005).

A. Salomon, T. Boecking, O. Seitz, T. Markus, F. Amy, C. Chan, W. Zhao, D. Cahen, and A. Kahn, Advanced Materials 19, 445 (2007); L. Segev, A. Salomon, A. Natan, D. Cahen, L. Kronik, F. Amy, C. K. Chan, and A. Kahn, Physical Review B 74, 165323 (2006); O. Seitz, T. Bocking, A. Salomon, J. J. Gooding, and D. Cahen, Langmuir 22, 6915 (2006); O. Seitz, A. Vilan, H. Cohen, C. Chan, J. Hwang, A. Kahn, and D. Cahen, Journal of the American Chemical Society 129, 7494 (2007).

7 A. Salomon, T. Boecking, C. K. Chan, F. Amy, O. Girshevitz, D. Cahen, and A. Kahn, Physical Review Letters 95, 266807 (2005). 
A. Salomon, D. Cahen, S. Lindsay, J. Tomfohr, V. B. Engelkes, and C. D. Frisbie, Advanced Materials 15, 1881 (2003).

9 P. Fontaine, D. Goguenheim, D. Deresmes, D. Vuillaume, M. Garet, and F. Rondelez, Applied Physics Letters 62, 2256 (1993).

10 D. K. Aswal, S. Lenfant, D. Guerin, J. V. Yakhmi, and D. Vuillaume, Analytica Chimica Acta 568, 84 (2006).

11 J. Y. Park, D. F. Ogletree, M. Salmeron, C. J. Jenks, and P. A. Thiel, Tribology Letters 17, 629 (2004); J. Y. Park, D. F. Ogletree, M. Salmeron, R. A. Ribeiro, P. C. Canfield, C. J. Jenks, and P. A. Thiel, Physical Review B 74, 024203 (2006). J. A. Dagata, J. Schneir, H. H. Harary, C. J. Evans, M. T. Postek, and J. Bennett, Applied Physics Letters 56, 2001 (1990); H. C. Day and D. R. Allee, Applied Physics Letters 62, 2691 (1993).

13 L. Fang, J. Y. Park, H. Ma, A. K. Y. Jen, and M. Salmeron, Langmuir 23, 11522 (2007).

14 Y. Qi, I. Ratera, J. Y. Park, P. D. Ashby, S. Y. Quek, J. B. Neaton, and M. Salmeron, Langmuir 24, 2219 (2008).

15 J. E. Sader, J. W. M. Chon, and P. Mulvaney, Review of Scientific Instruments 70, 3967 (1999).

16 D. F. Ogletree, R. W. Carpick, and M. Salmeron, Review of Scientific Instruments 67, 3298 (1996). S. M. Sze, Physics of Semiconductor Devices (Wiley, New York, 1981). J. Y. Park, R. J. Phaneuf, D. F. Ogletree, and M. Salmeron, Applied Physics Letters 86, 172105 (2005). 

3745 (2002).

20 K. L. Johnson, K. Kendall, and A. D. Roberts, Proceedings of the Royal Society of London Series a-Mathematical and Physical Sciences 324, 301 (1971).

21 The exact estimation of contact area requires consideration of elastic deformation of thin film. .

22 J. Y. Park, Y. B. Qi, I. Ratera, and M. Salmeron, Journal of Chemical Physics 128, $4(2008)$.

23 J. W. Zhao and K. Uosaki, Journal of Physical Chemistry B 108, 17129 (2004).

24 K. Slowinski, R. V. Chamberlain, C. J. Miller, and M. Majda, Journal of the American Chemical Society 119, 11910 (1997). 


\section{Figure Captions}

Figure 1. (Color online) Schematic of AFM measurement of current and friction on selfassembled monolayer organic molecules / Si (111) surface. The image in the schematic is the AFM image (400 nm x $500 \mathrm{~nm}$ ) taken in the contact mode AFM.

Figure 2. (Color online) $500 \mathrm{~nm}$ x $500 \mathrm{~nm}$ images of (a) topography (b) friction, (c) current taken in contact AFM mode on hexadecylsilane molecules / Si (111) surface. (applied load $=0 \mathrm{nN}$ or effective load of $20 \mathrm{nN}, \mathrm{V}=-2 \mathrm{~V}$ ) Line profiles of (d) topography (e) friction, (f) current revealing the low friction and low current on the molecular islands between A and B, shown in (a) .

Figure 3. (Color online) Change of current-voltage curves, measured on hexadecylsilane islands, as a function of the applied load. An I-V curve measured on the silicon surface at the applied load of $0 \mathrm{nN}$ is also shown.

Figure 4. (Color online) (a) Friction and (b) current measured as a function of the applied load on measured on hexadecylsilane $(\mathrm{C} 16)$ islands and silicon surface $(\mathrm{V}=$ $0.8 \mathrm{~V})$. The inset shows the friction and current maps as a function of the applied load (x axis). While the current on silicon surface was obtained from the inset, the current data on hexadecylsilane islands shown in (b) were taken at $-0.8 \mathrm{~V}$ (forward bias) from each I-V curves at various loads. 
Figure 5. (Color online) Semilog plot of current density $\left(\mathrm{nA} / \mathrm{nm}^{2}\right)$ as a function of the height of the molecules. The current values were taken at $-0.8 \mathrm{~V}$ (forward bias) from each I-V curve at various loads. A decay constant $\beta=0.52 \pm 0.04 \AA^{-1}$ was found for the current passing through the film as a function of tip-substrate separation. The solid line shows the fit of a two-pathway conductance model involving tunneling along the length of the alkyl chain (through-bond), and also chain-to-chain coupling. Fitting parameters of $\beta_{T B}\left(0.9 \AA^{-1}\right)$ and $\beta_{T S}\left(1.1 \AA^{-1}\right)$ were used. 
Conductive probe atomic/friction force microscopy

Alkylsilane SAM island

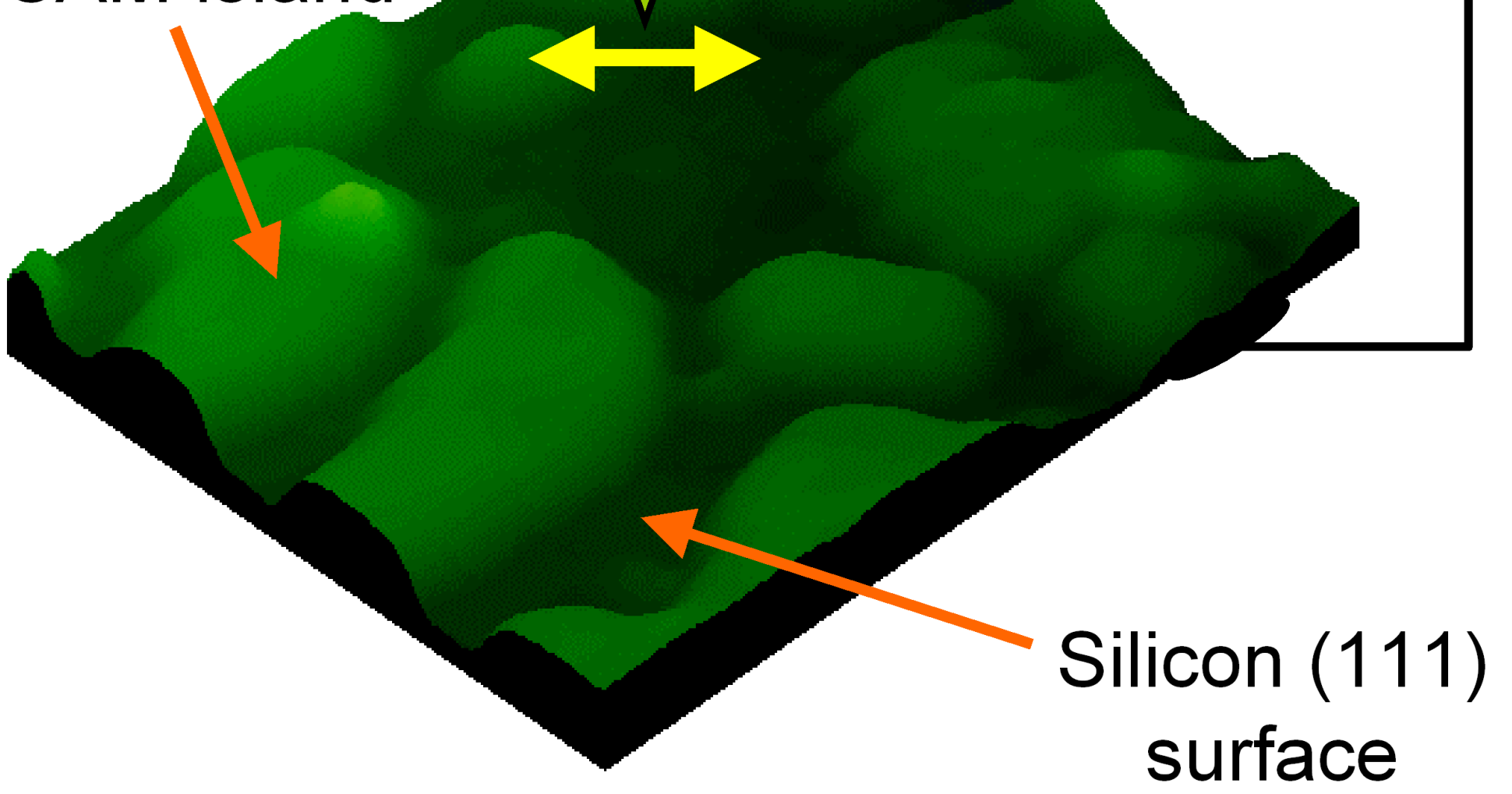



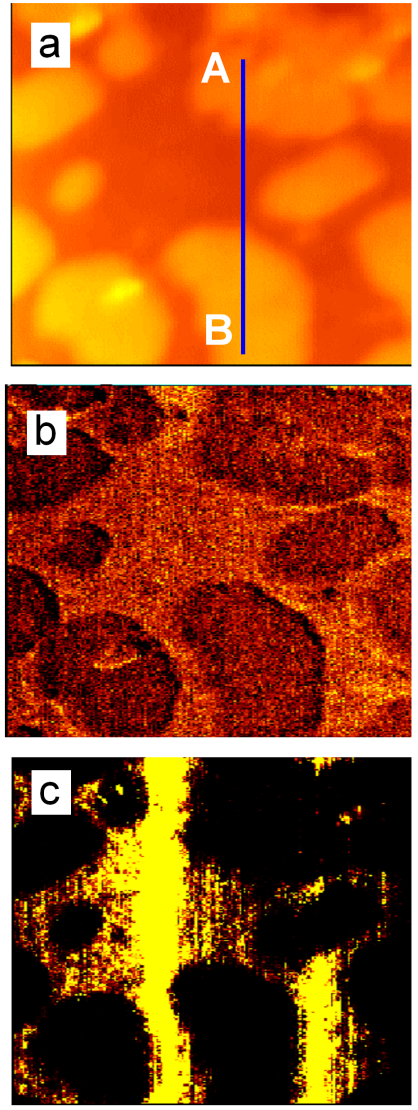
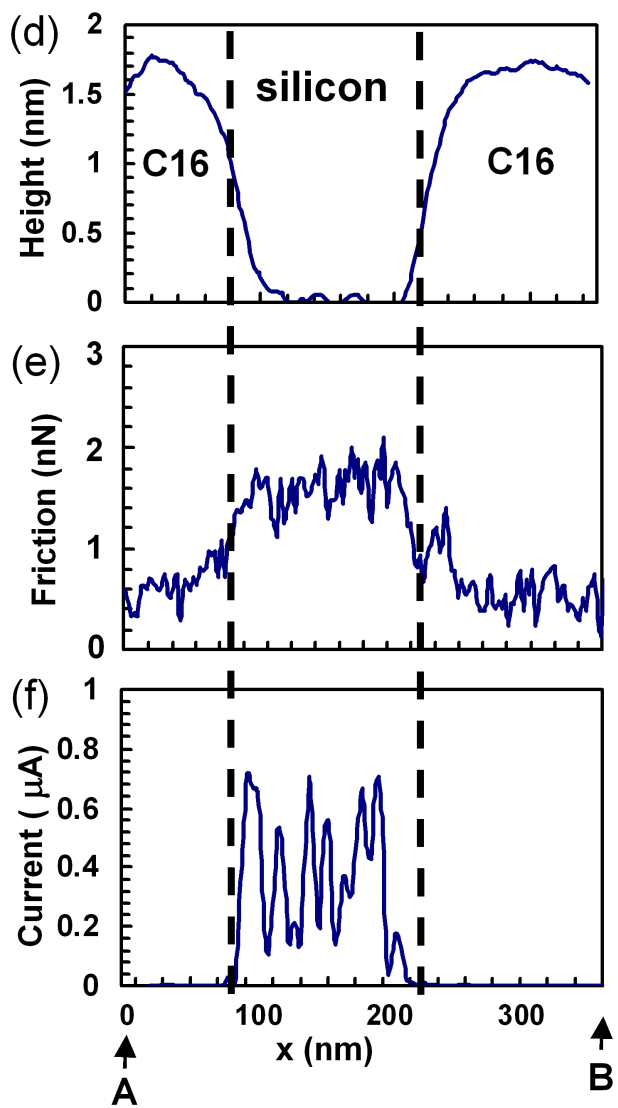


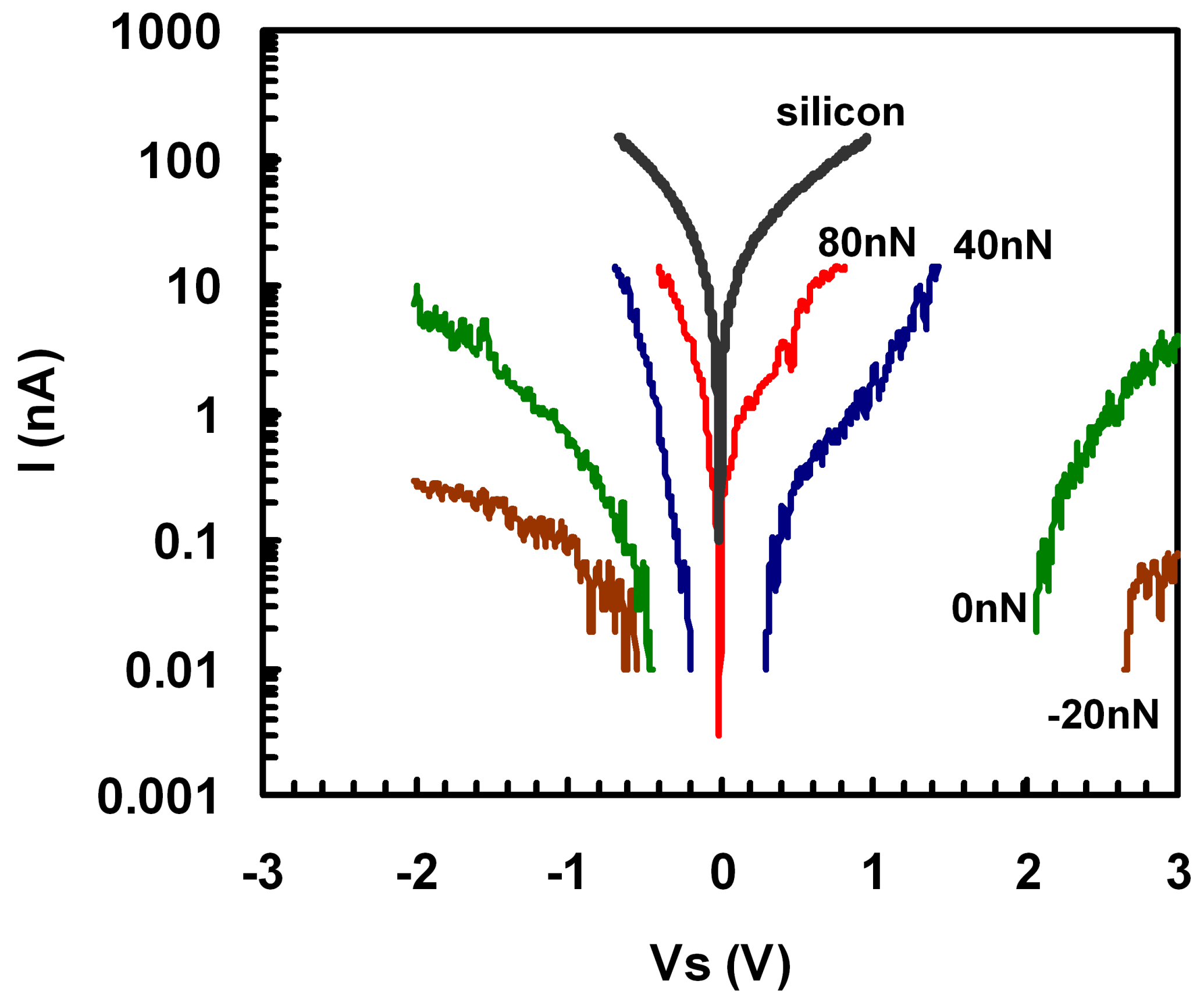




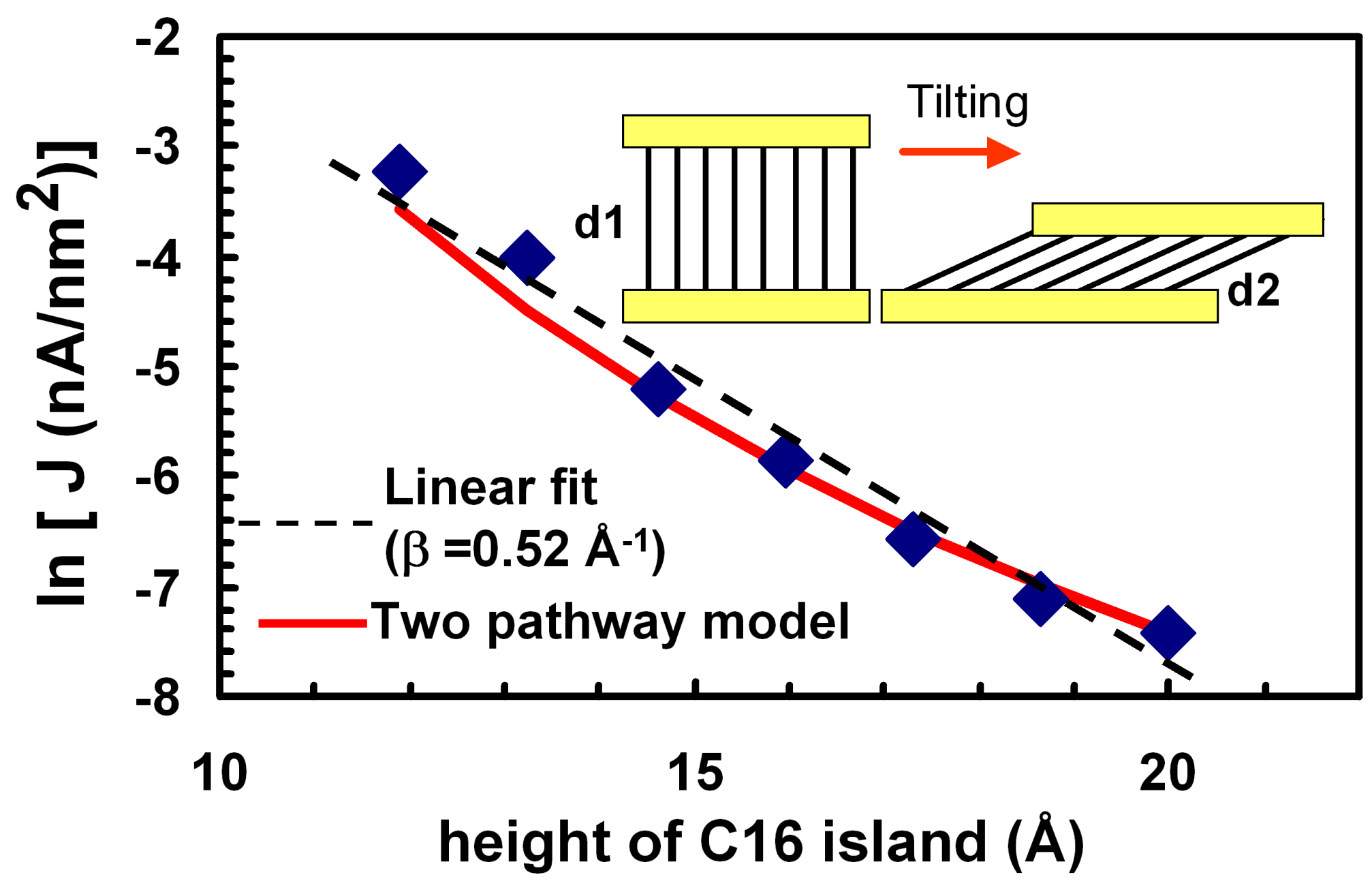

